\title{
Tumor Identifier Evaluator Identifier
}

National Cancer Institute

\section{Source}

National Cancer Institute. Tumor Identifier Evaluator Identifier. NCI Thesaurus. Code C117433.

A sequence of characters used to identify, name, or characterize the evaluator of the tumor identification. 